J. Andersen (ed.), Reports on Astronomy, Vol. XXIVA, 48-59.

(C)2000 IAU. Printed in the United States of America.

\title{
COMMISSION 24: PHOTOGRAPHIC ASTROMETRY
}

\section{(ASTROMETRIE PHOTOGRAPHIQUE)}

\author{
PRESIDENT: E. Schilbach \\ VICE-PRESIDENT: Wenjing Jin \\ ORGANIZING COMMITTEE: M. Crézé, P. D. Hemenway, I. I. Kumkova, \\ I. K. Platais, S. Röser, C. Turon \& J. J. Wang
}

\section{INTRODUCTION}

The scope of scientific interests of about 130 Commission members is much more complex than one may expect from the title of the commission. Besides traditional topics like the compilation of astrometric catalogues and the construction of an inertial reference system, more and more investigations performed by our members have been dedicated to the astrophysical interpretation of observations including among others astrometric data.

Nevertheless, photographic observations play a significant role in the scientific activities of many members of Commission 24. So far, digitized photographic plates have been used as the only observational method to provide deep optical surveys of positions and, especially, to determine proper motions of large numbers of stars fainter than $V=13$. These data are necessary for investigations of the Galactic structure and kinematics, studies of open and globular clusters, the search for low-mass nearby stars, and so on.

However, photographic observations are not the only source of astrometric information used by members of our Commission. Nowadays, astrometric data are obtained with CCD detectors, from radio, speckle and interferometric observations. Our members contributed considerably to the success of the Hipparcos mission, and some have been forming scientific teams which have proposed and developed future space astrometric projects like SIM, GAIA, FAME and DIVA.

Due to the new observational techniques and methods, a clear separation of activities between Commissions 8 (Positional Astronomy) and 24 is rather difficult. Taking also into account the significant number of common membership, a merger of both Commissions appears to be quite a logical solution. Three years ago, the corresponding proposal was distributed among the members of both Commissions and was supported by the vast majority. The merger will be effected at the XXIVth General Assembly in the year 2000.

To avoid repetitions in the reports of Commissions 8 and 24, we tried to separate the items as far as possible. Therefore, for a complete overview of the subjects related to astrometry, the reader is also referred to the reports of Commission 8 and of the Working Groups of Division 1.

\section{SCIENTIFIC HIGHLIGHTS}

The most important event of the past three years was the release of the Hipparcos and Tycho Catalogues in 1997. With some 1000 published papers based on Hipparcos data, the influence of accurate astrometric data on practically every field in astronomy has become obvious. For global astrometry the Hipparcos mission clearly demonstrated the superiority of observations from space and proves that proposals for follow-on missions are very attractive and appropriate. C. Turon, member of the Hipparcos Science Team and leader of the INCA Concortium (1981-1997), reports on behalf of the Hipparcos community: 


\section{Hipparcos Catalogue: publication and applications}

The characteristics of the Hipparcos Catalogue have been described in the report 1993-1996 of Commission 24. The years 1996 and 1997 have been devoted to prepare the publication of the Catalogue: multiple tests of the data (internal and external consistency, zero points, cross-check of related data), preparation of the documentation (description of the data, of the methods used to obtain them, of the calibrations, of the satellite, of the operations etc.). The Hipparcos and Tycho Catalogues (17 volumes) was published in June 1997. Many institutes in Europe were deeply involved in the Hipparcos data reduction and catalogue publication (ESA, CERGA (Grasse), Lund Observatory, Institute of Astronomy (Cambridge), SRON (Utrecht), Paris-Meudon Observatory, Genève Observatory, ARI (Heidelberg), etc.) and many others contributed to this long-term cooperative achievement. Three volumes of the Catalogue are the result of a remarkable collaboration between ESA, the Hipparcos teams and Sky Publishing Corporation: the Millenium Star Atlas, showing the stellar objects observed by Hipparcos along with non-stellar objects, galaxies and nebulae.

In parallel, the publication of a dedicated CD-ROM, Celestia 2000, released in April 1998 was also prepared. It includes the Hipparcos and Tycho Main Catalogues, the Hipparcos Double and Multiple Systems Annex, the Hipparcos Variability Annex, and complementary ground-based data from the updated Hipparcos Input Catalogue and its Double and Multiple Stars Annex. Celestia 2000 gives easy access to the data of each catalogue and annex, including notes and references, identification charts, charts of systems resolved by Hipparcos, and variable star light curves, if any. The software offers a wide range of possibilities, described in full detail in the on-line help facility, which also includes the full Hipparcos and Tycho Catalogue documentation. The Observatoire de Paris-Meudon Hipparcos group was the coordinator of and the main contributor to this CD-ROM.

A major symposium, presenting the final results of the mission and the first scientific applications in many fields of astrophysics was held in Venice on 13-16 May 1997 under ESA's auspices. 300 scientists met on the small island of San Giorgio, and the 800-page proceedings were published by ESA in July 1997 as ESA SP-402.

Giving the distances of more than 20000 stars (some as far as $200 \mathrm{pc}$ ) with an accuracy of better than $10 \%$, the distances of another 30000 stars better than $20 \%$, and a parallel progress in the accuracy of proper motions, the Hipparcos Catalogue provides an enormous new input for our understanding of the stellar component of the Galaxy. About 1000 papers making use of the Hipparcos data have been published since 1997. They deal with a high variety of subjects: reference systems; determination of orbits, masses and photometry of solar system objects; stellar physics: calibration of absolute luminosities and detailed studies of the HR diagram, stellar ages and evolution, test of asteroseismic analysis, stellar masses; census of the solar neighbourhood, with 200 'new' nearby stars (lying within $25 \mathrm{pc}$ ) and a few hundreds demonstrated to be much further away (reducing the number of giants within 25 pc by almost a factor two), 37 previously unknown binary companions; galactic physics: first unambiguous and accurate mean distance, 3-D structure and kinematic description of the Hyades; accurate mean distances for the nearest open clusters; accurate kinematic description of the associations; determination of the local mass density showing that the dark matter needed to explain the flat rotation curve of the Galaxy is not in the form of a disc; etc.

Perhaps one of the most spectacular result provided by Hipparcos are the steps towards solving the long-standing perturbing inconsistency that the expansion age of the Universe appeared to be smaller than the age of the oldest objects in our Galaxy, subdwarf stars and globular clusters. New absolute magnitude calibrations of some distance indicators tend to show that the older subdwarfs and globular clusters would be younger (ages about to 12-14 Gyrs) compared to the findings of pre-Hipparcos investigations. 


\section{SPECIAL PROJECTS}

\subsection{Positions and proper motions}

\section{Catalogues and Observing Programmes}

The Tycho observations from the ESA satellite Hipparcos have provided astrometry and photometry for about one million stars in the Tycho-1 Catalogue, published in 1997. Based on Tycho-1 and Astrographic Catalogue positions but on independent reduction of observations, two teams, at the US Naval Observatory (USNO) and at the Copenhagen University Observatory in cooperation with Heidelberg, Lund and Moscow, constructed two versions of the catalogue, i.e. the ACT Reference Catalog and the Tycho Reference Catalogue (TRC), respectively.

Gauss, at USNO, reports on the reduction of all Astrographic Catalogue (AC) zones to the Hipparcos system. The resulting catalogue, the AC 2000, contains positions of 4.6 million stars at an average epoch of 1905 . Standard deviations per image range from 150 mas to 450 mas. The AC 2000 was combined with the Tycho positions to derive proper motions for about 1 million Tycho stars. The resulting catalogue, the ACT Reference Catalog, has proper motion errors of 2 to 3 mas/year, about an order of magnitude better than the proper motions given in the Tycho catalogue. Both the AC 2000 and ACT Reference Catalog have been distributed on CD-ROM by the USNO.

$\mathrm{Høg}$, at Copenhagen, and Kuzmin, at Moscow, report on the construction of the TRC which contains high-quality positions and proper motions for 990182 stars and is available from CDS. The proper motions were derived from Tycho-1 positions and Astrographic Catalogue positions reduced to the Hipparcos system. The median accuracy of the TRC position components is 40 mas at J1991.25. The median accuracy of the proper motions is about $2.2 \mathrm{mas} / \mathrm{yr}$. Systematic errors are less than $1.0 \mathrm{mas}$ (mas/yr). The quality of the proper motions in TRC has been assessed by comparisons with the Hipparcos and Tycho-1 Catalogues and with ACT Reference Catalog.

Both teams have been collaborating in producing the Tycho-2 Catalogue. Høg and Gauss report: The original Tycho photon counts from the satellite were re-reduced with more powerful computers and software by a team at Copenhagen, supported by colleagues at Heidelberg and Munich. The resulting Tycho-2 Catalogue shows improved astrometric and photometric precision of faint stars in the Tycho-1 Catalogue, and the total number of stars is increased to 2.5 million. The improvement in the new reduction compared to Tycho- 1 is achieved by making a superposition of the photons that were counted during all the about 130 observations per star obtained during the mission. USNO is responsible for computing the Tycho-2 proper motions. As a first step, USNO converted over 150 transit circle and astrograph catalogues to the system defined by Hipparcos. These positions were then combined to form a new reference catalogue only for the purpose of re-reducing the Astrographic Catalogue data. A new reduction of the AC measures was made, using the Tycho-2 photometry to remove systematic errors based on colour and magnitude. The AC, transit circle, astrograph, and Tycho-2 positions were then combined to form the Tycho-2 proper motions. The Tycho-2 Catalogue will contain positions and proper motions with median accuracies of respectively 60 mas and 2.5 mas $/ y r$. Two-colour photometry $\left(B_{T}, V_{T}\right)$ is also included. The accuracy of $V_{T}$ is $0.012 \mathrm{mag}$ for $V_{T}<9 \mathrm{mag}$ and the median accuracy is 0.09 mag. The catalogue is $\sim 99.9 \%$ complete to $V \sim 11.0 \mathrm{mag}$ and the limiting magnitude is $V \sim 12.5$ mag. Tycho- 2 will be available on CD-ROM and through CDS.

Gauss, reports on the modification of the USNO double astrograph from a photographic instrument to one equipped with a $4 \mathrm{k} \times 4 \mathrm{k}$ CCD camera. The blue lens was replaced by a new five-element red lens to be used with the CCD camera and the yellow lens used as the guide telescope. In January 1998 the USNO CCD Astrograph Catalog (UCAC) project started at Cerro Tololo (CTIO) Chile. After three years of observations, the astrometric survey will cover the sky from the South Celestial Pole to about +25 degrees declination, with a limiting magnitude of about 16 in a $579-642 \mathrm{~nm}$ bandpass. An accuracy of about 20 
mas is expected for stars in the 8 to $14 \mathrm{mag}$ range. An independent link to the extragalactic reference frame is part of the programme. By the end of June 1999, over 26,500 survey fields $(44 \%)$ were observed. When the Southern observations are complete the telescope will be moved to the northern hemisphere and the all-sky programme will be provided.

Girard, Platais and van Altena, at Yale, report on the Southern Proper Motion (SPM) Catalog 2.0 which is now completed and available on the website. The catalogue provides positions, absolute proper motions, and photographic BV photometry for over 320,000 stars and galaxies. It covers an area of 3700 square degrees in a declination band from -43 to -22 degrees, but excluding fields in the plane of the Milky Way. For the stars measured best, standard errors of $20 \mathrm{mas}, 2 \mathrm{mas} / \mathrm{yr}$ and $0.05 \mathrm{mag}$ were determined for positions, absolute proper motions and B, V magnitudes, respectively. In addition to the catalogue, an extensive list of CCD calibrating sequences in $\mathrm{B}$ and $\mathrm{V}$ is provided. Due to the lack of photographic plates, the remaining SPM second epochs are expected to be obtained with a PixelVision $4096 \times 4096$ CCD camera which covers about one square degree on Yale/San Juan double astrograph. Currently, all of the SPM photographic plates are being measured by D. Monet at Flagstaff with the PMM high-speed measuring machine. As a result, a complete census of absolute proper motions will be expected in the southern hemisphere down to about $\mathrm{V}=18.5$.

Klemola, Hanson, and Jones (Lick observatory) continued work on Part II of the Lick Northern Proper Motion (NPM) programme (350 Milky Way fields north of -23 degrees). The measurements, astrometric and photometric reductions of 61 NPM2 fields were finished. Hipparcos proper motions were used to link the NPM2 proper motions to the NPM1 extragalactic reference frame. Additional 83 fields were surveyed to select stars from the Lick Input Catalog of Special Stars, which has been expanded to nearly 300,000 entries. With the cooperation of D. Monet (USNO, Flagstaff), 3882 NPM plates, including all NPM1 and NPM2 fields, were scanned on USNO's PMM. After reduction, the PMM scans for several hundred thousand stars will form the basis for the NPM2 Catalog, on schedule to be published in 2002 .

Kislyuk, at Kiev, reports on the plate solutions of the Golosiiv part of the FONprogramme (photographic survey of the northern sky) which were finished in Kiev. The catalogue contains positions, proper motions, B-magnitudes and B-V, B-R colours of about 2 million $\mathrm{AC}$ stars at an average epoch 1988. The mean errors are 200 mas, 3 mas/year and $0.18 \mathrm{mag}$, respectively.

Rizvanov, at Engelhardt Observatory, reports on the catalogue of photographic positions in the SRC for 25,687 stars. The observations were carried out in 1979 with the Zeiss astrograph $(\mathrm{f}=2000 \mathrm{~mm}, \mathrm{D}=400 \mathrm{~mm})$ located at Zelenchuk. It is proposed that the data be used for the improvement of the catalogue FOCAT.

\section{Catalogue Compilations and Re-reductions}

Gauss, at USNO, reports on the re-reduction of the CPC2 data, based on Hipparcos reference stars. The CPC2 covers the Southern Hemisphere to a limiting magnitude of about $\mathrm{V}=10.5$ and contains positions of 274,669 stars with an average accuracy of 53 mas at the mean epoch of 1968 (Zacharias et al. 1999). New Hipparcos-based reductions of the Twin Astrograph Catalog (TAC) were also completed (Zacharias \& Zacharias 1999). The TAC contains positions stars, covering most of the sky between declinations +90 and -18 degrees to an average limiting magnitude of about $\mathrm{V}=11(\mathrm{~B}=12)$. Depending on magnitude, the accuracy ranges from 48 to 120 mas at the mean epoch of 1982 .

Yagudin, at Pulkovo Observatory, reports on the determination of proper motions in the FK5 system for about 4.3 million stars in a magnitude range from 8 to 12 , derived from the GSC++ (the Pulkovo/Valencia re-reduction of the GSC) and the AC (the Washington version). Whereas the random errors are about 2 to 4 mas/yr, systematic errors were estimated to be about two times larger. 


\section{Plate Measurements and Multi-Wavelength Cross-Identifications}

Guibert, at Centre d'Analyse des Images, Paris, reports that during the last three years, more than 1,500 Schmidt plates (ESO, SRC, Palomar, Tautenburg, OCA) have been digitized with the MAMA microdensitometer. The publication of the ACT allowed to reach an astrometric accuracy of $0.1^{\prime \prime}-0.2^{\prime \prime}$ using about $800-1000$ reference stars per field. Parts of the calibrated images (mainly in crowded regions such as the Bulge, Galactic Plane and the Magellanic Clouds) are already accessible through the ALADIN software at CDS. The complete data sets can be made available on request. MAMA plate measurements have been used in different programmes, e.g. galactic structure and cluster studies, for identification and determination of the positions of optical counterparts of sources detected at other wavelengths (from IR to X-rays), both in the galactic and extragalactic domains (variable stars, planetary nebulae in the LMC, clusters and super clusters of galaxies), as well as for the astrometric reduction of CCD images via reference catalogues constructed from Schmidt data.

Monet, at Flagstaff, reports on activities based on measurements with the PMM highspeed measuring machine which has been digitizing plates since November of 1994, and has already processed over 13 trillion pixels and logged more than 10 billion detections. The surveys completed include POSS-I O, E, and Whiteoak extension, PIMWA, POSS-II UJ, $\mathrm{J}, \mathrm{F}, \mathrm{N}$, and various rejected plates, ESO-R, SRC-J, SERC-EJ, most of SERC-ER, most of AAO-R (also known as SES), all of the Lick NPM plates, and about half of the Yale SPM plates. From some of these data, the USNO-A2.0 catalogue of 526,280,881 objects was compiled and distributed to the community. Projects for the near future include access to the pixel and detection databases, and the compilation of the USNO-B catalogue which will contain estimates for proper motion and star/galaxy classification.

Geffert, at Bonn, reports on the determination of positions for candidates of optical counterparts of X-ray sources in the fields of the globular clusters 47 Tuc, M3 and M92 (Geffert et al. 1997, Geffert 1998). Possible identifications of the central sources were discussed; for M92 corrected X-ray positions were determined.

Scholz, at Potsdam, reports on the combined variability-proper motion surveys for QSOs based on series of Tautenburg Schmidt plates taken during 30 years near the North Galactic Pole and around M 92. Using variability indices, a structure function analysis was applied to search for long-term variability. Proper motions were obtained with a mean error of about $2.0 \mathrm{mas} / \mathrm{yr}$ for $B<19.5$. At a limiting magnitude of $B_{\text {lim }}=19.7$ the completeness of the QSO sample is expected to be about 90 per cent. The final sample contains 168 QSO candidates; only one of them has a radio counterpart. Spectroscopic follow-up observations in both fields confirm the estimated high success rate (Scholz et al. 1997, Meusinger et al. 1998).

\subsection{Trigonometric Parallaxes and Nearby, Low-Mass, and High Proper Mo- tion Stars}

Jahreiß, at Heidelberg, reports on the extraordinary impact of the Hipparcos data on the Catalogue of Nearby Stars. The typical error of a trigonometric parallax in this catalogue dropped from 8 mas to 1.8 mas. Especially the bright portion $\left(M_{V}<8^{m}\right)$ of the catalogue was complemented and cleaned up by Hipparcos, i.e., the catalogue represents now the true distribution of the stars with masses larger than $0.6 m_{\odot}$ within $25 \mathrm{pc}$. A preliminary data base version of the Catalogue of Nearby Stars ARICNS is available on the website.

Ducourant, at Bordeaux, reports on the results of a search for nearby stars using Schmidt plates. In a field, intensively observed, a list of 32 nearby stars candidates was selected. CCD astrometry of 20 of them was obtained with the $1.23 \mathrm{~m}$ telescope of Calar Alto. Trigonometric parallaxes and proper motions were determined. For six targets, distances smaller than 40 parsecs (for three of them, smaller than 20 parsecs) were confirmed with relative errors less than 20 per cent (Ducourant et al. 1998). Further, a survey for low 
mass stars and brown dwarfs was started on the basis of a photographic stack of around 100 Schmidt plates. This survey extends photographic searches to about 2 magnitudes fainter, and covers an area of 25 square degrees. For 30 pre-selected faint objects, very strong molecular absorption in their spectra but only moderately red-infrared colours, were found. For 5 of them, the trigonometric parallaxes were determined. 3 objects were found to be located within $40 \mathrm{pc}$ from the Sun. On the basis of the luminosity alone it is clear that these objects are brown dwarfs (Hawkins et al. 1998).

Ianna, at Charlottesville, reports on the progress on the southern CCD parallax programme with the 1.0-m telescope at Siding Spring Observatory. In addition to the parallax observations, limited VRI photometry is done to select other low luminosity stars possibly within $20 \mathrm{pc}$. A list of parallaxes, including a number of stars within $10 \mathrm{pc}$, is in preparation. The nearby star programme has been expanded to CTIO (0.9 and $1.5 \mathrm{~m}$ telescopes) through a NOAO survey award (in cooperation with T. Henry, P. Seitzer and others). The aims of this programme are: to identify new nearby star candidates through photometry and to obtain parallaxes of the nearest ones. Precision will be limited to about 3 mas in order to quickly obtain results for a larger number of stars toward filling in the nearby star data base in support of the NASA NStars Project.

Monet, at Flagstaff, reports that, within the USNO Flagstaff Station parallax programme, new observations were obtained with the Tektronix $2048 \times 2048$ CCD (Tek2K) and ND9 sensors. The Tek2K has already produced about 200 parallaxes with a median error of $1.0 \mathrm{mas}$, and the first observations with the ND9 sensor (a SITe 2048x2048 CCD with a 9-magnitude occulting spot in proximity focus) indicate that similarly good astrometric results can be expected. The emphasis of the Tek2K programme is shifting to the recently discovered very faint $\mathrm{L}$ - and T-dwarfs found by the 2MASS and SDSS surveys. The next detectors to which the parallax programme will have access are ND5 sensor (a 5-magnitude occulting spot system) and a $1024 \times 1024$ InSb infrared array camera.

Scholz, at Potsdam, reports on a systematic search for high proper motion (HPM) stars in the southern sky on the basis of APM measurements of UKST survey plates (Scholz et al. 1999). In an area of about 1000 sq. degrees, some 100 stars with proper motions larger than $0.3 \mathrm{arcsec} / \mathrm{yr}$ were discovered, another 100 already known HPM stars were confirmed. The faintest discovered HPM stars are a common proper motion pair $\left(R_{1}=19.7, R_{2}=20.9\right.$, $\mu=0.5 \mathrm{arcsec} / \mathrm{yr})$; they are estimated to be extremely cool degenerate white dwarfs. For a sample of pre-selected new HPM stars, their nature as cool white dwarfs, late-type Mdwarfs or normal white dwarfs was confirmed by follow-up spectroscopy with the $1.9 \mathrm{~m}$ Radcliffe telescope at the SAAO (Scholz et al. 1999, Schweitzer et al. 1999). Additionally, a new active M5-dwarf $(R=13.4)$ was detected and identified with an $\mathrm{X}$-ray source. With a distance between 11 and $14.5 \mathrm{pc}$, this is a late-type M-dwarf with high X-ray luminosity. As a by-product of the proper motion study in the field of the globular cluster Pal 5 (Scholz et al. 1998), a new HPM cool subdwarf $(R \approx 18)$ was discovered. Spectroscopy indicates that it is a cool metal-poor star with spectral type sdM5.5, i.e. only the sixth known subdwarf later than type sdM5.0 (Gizis et al. 1997).

Girard, Wu, Lee, Dyson, Horch, and van Altena, at Yale, report on the re-determination of astrometric orbit and parallax of the Procyon system based on Yale PDS measures of plate series from McCormick, Sproul, USNO, Van Vleck, Yale, and and Yerkes Observatories. The parallax, $283.2 \pm 1.5$ mas, differs only slightly from the published value, but the orbital parameters disagree somewhat with those found before. The resulting derived mass of Procyon A now comes into agreement with estimates deduced from stellar evolution models, reconciling a longstanding disagreement between observation and theory concerning this star.

Geffert, at Bonn, reports on the determination of galactic orbits and distribution perpendicular to the Galactic plane of 40 blue subdwarf stars (de Boer et al. 1997).

Fresneau, at Strasbourg, investigated the possibility for the search of low-mass stars in the Gould's Belt system by analysing the kinematics of stars in this region. The kinematic 
parameters were derived from proper motions by the use of the $\mathrm{AC}$ as the first epoch positions (Fresneau 1996).

\subsection{The Extragalactic Reference Frame}

For the final extragalactic link of the Hipparcos proper motion system, many photographic multi-epoch observations were used. The details of the correspondings programmes at Bonn, Lick, Kiev, Potsdam, and Yale were already described in the report 1993-1996 of Commission 24 (see also Kovalevsky et al. 1997).

Jin, at Shanghai, reports on new positions of 31 radio stars obtained with the 40$\mathrm{cm}$ astrograph at $Z \hat{o}-S e ́$ Section of the Shanghai Astronomical Observatory (Lu \& Xu 1997). The positions of four radio sources in the Hipparcos system were derived from CCD observations with $60 / 90 \mathrm{~cm}$ Schmidt telescope of the Beijing Astronomical Observatory (Tang et al. 1998).

Rizvanov, at Engelhardt Observatory, reports on a catalogue of photographic positions of about 20,000 stars with magnitudes between 13 and 17 obtained with the Zeiss astrograph at Zelenchuk. The observations were carried out in fields around 190 extragalactic radio sources (ERSs). This catalogue is used as a reference for the reduction of CCDbased observations of the ERSs. Additionally, positions of 132 galactic radio sources were determined with the same instrument.

For details on this chapter, see reports of Commission 8 and of the Working Group on the International Celestial Reference System.

\subsection{Galaxy, Open and Globular Clusters}

\section{Open Clusters}

Geffert, at Bonn, reports on the determination of the luminosity function for the open cluster NGC 581 based on the combination of photographic proper motions and CCD photometry (Sanner et al. 1998).

Kharchenko, at Kiev, and Schilbach, at Potsdam, report on a study of the luminosity (LF) and mass (MF) functions of eight open clusters in the directions of both the Galactic centre and anticentre. The study was based on MAMA measurements of Tautenburg Schmidt plates. The composite MF was constructed and a possible relation between the individual MF slopes and cluster ages was indicated (Kharchenko et al. 1998). The Tautenburg survey of the extremely young open cluster NGC 6611 was supplemented by photometric and proper motion data published in this region which were reduced to a common homogeneous system. The fine structure of the LF was analysed and a detail in the cluster's LF was detected which, according to theoretical predictions, should appear due to the presence of pre-MS stars and can be used as an independent age indicator (Belikov et al. 1999). A similar study was also carried out for the Pleiades (Belikov et al. 1998).

Platais, Girard, and van Altena, at Yale, report on a programme of deep astrometry in six selected open clusters (NGC 188, 2264, 2451, 2682, 6791 and 6819) which will provide cluster memberships of at least six magnitudes fainter than the existing studies of this kind. This has been enabled by the use of KPNO 4-m telescope archival photographic plates in combination with the current-epoch CCD Mosaic Imager on the same telescope. The programme will provide high precision data for open clusters in a wide range of ages and metallicities.

Scholz, at Potsdam, reports on a proper motion survey down to $R=18$ in a one square degree region around the young open cluster IC 348. According to membership probabilities, 240 cluster candidates were separated, and a cluster radius of $10-15$ arcmin was determined. High membership probabilities were derived for the identified ROSAT X-ray sources. They are likely T Tauri stars and belong to the cluster IC 348 which is embedded in the Per OB 2 association (Scholz et al. 1999). 
Jin and Wang, at Shanghai, report on an open cluster programme based on astrometric and photographic data obtained from series of plates taken with the SHAO astrograph during 80 years. Proper motions and membership probabilities were determined for stars in the regions with the open clusters M 11,42, 67, NGC 1750, 1758, 1808, 1817 (Su et al. 1998, Tian et al. 1996, 1998 , Zhao et al. 1996). A possible mass segregation was found for M 11 and M 67. The proper motion data for M 42, also supported by spectroscopic and radial velocity measurements, indicate that the stars in $\mathrm{a} \approx 3 \mathrm{sq}$. degree region around the cluster are not bound into one system but have a common origin. Further, a distance of $135.6 \pm 0.7 p c$ (dispersion: $7.7 \pm 0.8 p c$ ) and space velocity of $25.9 \pm 0.13 \mathrm{~km} / \mathrm{s}$ (dispersion: $0.6 \pm 0.1 \mathrm{~km} / \mathrm{s}$ ) were determined for the Pleiades (Chen and Zhao 1997). For about 35 per cent of the Pleiades red dwarfs, no flare activity could be found (Wang et al. 1996).

\section{Globular Clusters}

Brosche and Geffert, at Bonn, report that in cooperation with Bordeaux galactic orbits of globular clusters with known absolute proper motions were determined (Dauphole et al. 1996). Among them, very eccentric orbits (median 0.6) have also been found. The tidal radii determined are not perigalactic ones but correspond to a certain average over the orbit.

Dinescu, Girard, van Altena, at Yale, used the SPM plates to determine absolute proper motions for 15 southern globular clusters spanning a Galactocentric radius in the range 3 to $15 \mathrm{kpc}$. It was found that a metal poor and old globular cluster NGC 6752 has a thick-disk-like kinematics - only the third globular of that kind. The second-parameter type clusters were found to have eccentric, highly energetic orbits, with radial anisotropy of the velocity dispersion. Up to the Galactocentric radius of $15 \mathrm{kpc}$, the new data and orbit calculations indicate very little, if any, evidence for a structure in the globular cluster phase-space characteristics (Dinescu et al. 1997, 1999).

Scholz, at Potsdam, reports on absolute proper motions of globular clusters derived from series of digitized Schmidt plates as deep as $B \approx 19$. Using the method of automated star counts, an indication was found for a tidal tail around NGC 5466, M 5, M 12, M 13 and M 15 (Lehmann et al. 1997). In the case of M 5, this effect was not confirmed by the astrometric member counts (Kharchenko et al. 1997). For the Galactic globular cluster Pal 5, the result suggests a total space velocity significantly different from one that were to be expected from a tidally stripped fragment of the Sgr dwarf galaxy. Moreover, Pal 5 was found to move almost in the opposite direction to that of the Sgr galaxy (Scholz et al. 1998).

Jin and Wang, at Shanghai, report on the absolute proper motion and BVRI photometry of the globular cluster NGC 4147 obtained with astrographic plates at Shanghai and CCD observations with the Vainu Bappu Telescope at Kavalur, India. The data were used to derive the space motion and the colour-magnitude diagram for NGC 4147 (Wang and Gupta 1998).

\section{Double and Variable Stars; Miscellaneous}

Fresneau, at Strasbourg, used deep plates of the Astrographic Programme to detect a possible variability of the interstellar extinction in the Vulpecula Rift (Fresneau 1999).

Kharchenko, at Kiev, and Schilbach, at Potsdam, report on a program package for the prediction of the numbers of stars of different types which can be expected down to a given limiting magnitude and in a given direction of the sky. The predictions are based on an improved three-component model of the Galaxy deduced from astrometric and photometric data of about 60,000 stars in the main galactic meridian and were used for the specification of the observing strategy for the survey with the space mission DIVA (Kharchenko et al. 1998). 
Dommanget and Nys (Royal Observatory, Belgium) have pursued the completion of the Catalogue of the Components of Double and Multiple systems (CCDM) which at present contains about 45.000 perfectly identified systems including the 2.994 resolved and 155 astrometric new pairs discovered by Hipparcos.

Falin and Mignard, at Grasse, re-processed the original Hipparcos data for stars which yielded unreliable astrometric solutions and was indicated as suspected double/multiple systems. By including also ground-based data, new values for the astrometric parameters as well as for the double star structure were proposed for more than 100 stars.

Kharchenko, at Kiev, and Schilbach, at Potsdam, analysed the complete data set on Mira variables from the GCVS and supplemented it by proper motions, reduced to the Hipparcos system, and radial velocities available for these Miras until now. The statistical parallaxes determined with five different methods, mean absolute magnitudes, spatial velocities and elements of the Galactic orbits were computed as functions of the periods.

Jin and Wang, at Shanghai, report on the determination of the space motions of Galactic field RR Lyrae stars based on their absolute proper motions (Wang et al. 1997).

\subsection{Instrumentation and Reduction Methods}

Kanaev, Deviatkin and Kulish, at Pulkovo Observatory, report that a new telescope, "Mirror Astrograph - 320", equipped with a CCD-camera became operational in 1997. According to preliminary test observations, an astrometric accuracy of 0.04 arcsec is expected at zenith distances less than 50 degrees. Observing programmes have been started and include regular observations of asteroids, satellites of Jupiter and Saturn, multiple stellar systems, UBV and UPXYZV photometry.

Jin, at Shanghai, reports on test observations with the Low Latitude Meridian Circle, which were carried in 1998 and yielded an accuracy af 0.14 arcsec for a single observation (Mao 1997). Once equipped with a CCD camera, the instrument will reach stars down to 17.5. Further, a new 105-cm Cassegrain telescope providing a scale of $20^{\prime \prime} / \mathrm{mm}$ was installed at Shaanxi Observatory in 1996. The performance of the telescope was tested at observations of comet Hale-Bopp and was found to be very satisfactorily (Yang et al. 1998).

Brosche, at Bonn, reports on his findings that with precise measurements and sophisticated complex reduction of Carte du Ciel plates, an accuracy of about $0.15^{\prime \prime}$ and $0.12^{m}$ can be achieved for positions and magnitudes, respectively (Ortiz-Gil et al. 1998). Geffert and co-authors from Bonn and Paris tested the accuracy of Carte du Ciel plates using old and new observations of the open cluster NGC 1647 (Geffert et al. 1996).

Deviatkin, at Pulkovo observatory, reports on a new program package for reduction of CCD images. This software takes into account chromatic aberration and allows astrometric processing in the system of the Hipparcos, Tycho and USNO catalogues.

Kovalevsky, at Grasse, discussed the relationship between parallax and distance, and showed that $r=1 / p$ is not the best estimate for distances.

D.J. MacConnell, R. L. Williamson II, and J. Roberts, at STScI, derived a procedure for determining proper motions using the two epochs of Palomar Sky survey for stars with motions $0.03 \leq \mu \leq 0.2^{\prime \prime} / y r$ and $V \geq 11.0$. This method was applied to T. Beer's weakmetal stars and for about 300 of them new proper motions were determined.

\subsection{Space astrometry}

The reports provided by members of Commission 24 on projects for astrometric space missions have been combined with those sent to Commission 8 and can be found in the report of Commission 8. 


\section{WORKING GROUP ON AC AND CDC PLATES}

The Working Group (WG) was established by the Commissions 8 and 24 in 1994 to evaluate the present state of the 100-years old photographic plates of the Astrographic Catalogue (AC) and Carte du Ciel (CdC) programmes, in order to prove the necessity of the measurement or re-measurement of the plates and to work out recommendations on the scientific use of one-century old stellar positions and apparent magnitudes in consideration of the overall plate quality. The WG includes about 40 members. A. Fresneau, Chairman, reports on the activities of the WG:

Presently, the only source of AC positions in the Hipparcos system is the AC 2000. These positions are also well suited to calibrate deep CdC plates since they were taken with the same telescopes and the same type of photographic emulsion was used.

The original goal of the Astrographic Programme proposed about 100 years ago can be now realized because most of the deep $\mathrm{CdC}$ plates are still available, though, at different observatories the plate vaults have been closed for 30 years. To provide scientific information stored on these plates to the community, two possibilities have been identified:

- to build a mobile measuring machine and to travel from a plate vault to a plate vault (Hiesgen et al. 1997) or

- to take the plates to an existing measuring machine being capable to handle a large number of plates in a relatively short time.

The WG is aware of the project for a mobile measuring machine and would prefer this solution. Nevertheless, due to limited resources, the second possibility was identified to have more chances for a practical realization.

The past two decades have proven that suitable measuring machines can digitize a set of some thousands of Schmidt plates and provide catalogues of star positions and magnitudes with epochs later than 1950 and 1970 for the northern and southern hemispheres, respectively (van Altena et al. 1997). With the CdC plates, the baseline for proper motion determination, especially for the southern hemisphere, can be considerably extended. Test measurements of a few tens of CdC plates in the southern zones of the Astrographic Programme are presently under way with different measuring machines:

- plates of the Cordoba Observatory with MAMA in Paris

- plates of the Cape of Good Hope Observatory with StarScan in Washington D.C.

- plates of the Sydney Observatory with APM in Cambridge (England)

- plates of the San Fernando Observatory with a PDS in Merida (Venezuela).

Additionally, test measurements of $\mathrm{CdC}$ plates of the Bordeaux zone are now carried out with MAMA (Paris), APM (Cambridge), and PMM (Flagstaff). These tests will not only provide a check of the overall astrometric and photometric quality of the data but also estimate the efforts needed for a routine measurement and reduction, and help to identify the measuring machines willing to handle a large set of $\mathrm{CdC}$ plates within a tightly focused scientific programme.

Some $\mathrm{CdC}$ plates were already used in different scientific programmes. The results demonstrated that a positional accuracy of about $0.15^{\prime \prime}$ is possible but a sophisticated complex reduction of the measurements is required (e.g. Brosche 1996, Geffert et al. 1996, Ortiz-Gil et al. 1998). Different programmes of plate modelling tested in Hamburg have shown that an accuracy of $1 \mu \mathrm{m}$ is achievable which can lead to an accuracy of proper motions of $1 \mathrm{mas} / \mathrm{yr}$. This accuracy is mandatory for a competitive astrometry. However, this is a big concern for the WG, because the quality of CdC plates is far from being excellent in many zones. Reasons are: radial and lateral aberrations outside a central field of 1 degree, grid lines every $5 \mathrm{~mm}$, triple images. These effects make an automatic reduction of digitized measurements rather difficult. Therefore, special software for analyzing scanned data is required, adapted to the needs of the specific measuring machines. 
As the first step, the WG considers that a trade-off has to be made between the final accuracy and the fast reduction of scanned raw data of the $\mathrm{CdC}$ plates to provide the astronomical community with the possibility of using these data in at least some selected regions (e.g., the Galactic plane, the ecliptic, open and globular clusters). A direct access to information on the plate collection is provided by the Toulouse observatory via Internet.

In conclusion, the old $\mathrm{AC}$ data have already demonstrated their scientific potential providing the basis for the TRC and ACT proper motions of 1 million stars. Taking into account that the modern instruments such as La Palma, Bordeaux, San Fernando (El Leoncito), and USNO (CTIO and Flagstaff) are capable of making precise astrometric measurements down to 15 th or 16 th magnitude, the $\mathrm{CdC}$ data are more important than ever. Investigations of the stellar kinematics up to a distance of $1 \mathrm{kpc}$ from the Sun appears quite possible with these plates.

The WG will prepare a draft resolution concerning the scientific legacy and potential of the 100-yr old astrographic plates as well as recommendations of their effective use which will be submitted to Commission 24 for discussion and adoption.

\section{SYMPOSIA, COLLOQUIA, CONFERENCES}

IAU Symposium 179, New Horizons from Multi-Wavelength Sky Surveys, Baltimore, USA, August 1996, B.J. Mc.Lean, D.A. Golombek, J.E. Hayes, H.E. Payne (eds), Kluwer.

ESA Symposium, Hipparcos-Venice '97, Venice, Italy, May 1997, B. Battrick, M.A.C. Perryman, P.L. Bernacca, K.S. O'Flaherty (eds), ESA-SP-402.

IAU Colloquium 165, Dynamics and Astrometry of Natural and Artificial Celestial Bodies, Poznan, Poland, July 1996, I. Wytrzyszczak, J.H. Lieske, R.A. Feldman (eds), Kluwer.

IAU Colloquium 176, The Impact of Large-Scale Surveys on Pulsating Star Research, Budapest, Hungary, August 1999 (in preparation).

IAU Colloquium 178, Polar Motion: Historical and Scientific Problems, Cagliari, Italy, September 1999 (in preparation).

IV International Workshop, Positional Astronomy and Celestial Mechanics, Peniscola, Spain, October 1996, A. Lopez Garcia et al. (eds)., Astron. Observatory, University of Valencia.

International Spring Meeting of the Astronomische Gesellschaft, The Message of the Angles - Astrometry from 1798 to 1998, Gotha, Germany, May 1998, P. Brosche, W.R. Dick, O. Schwarz, R. Wielen (eds), Verlag Harri Deutsch.

International Workshop, Treasure-Hunting in Astronomical Plate Archives, Sonneberg, Germany, March 1999 (in preparation).

Working on the Fringe: An International Conference on Optical and IR Interferometry from Ground and Space, Dana Point CA, USA, May 1999 (in preparation).

International Workshop, Nearby Stars, NASA-Ames, USA, June 1999 (in preparation).

Journees 1997 systemes de reference spatio-temporels. Reference systems and frames in the space era: present and future astrometric programmes, Prag, Czech Republic, September 1997, J. Vondrak \& N. Capitaine (eds.), Astron. Inst. Prague and Observatoire de Paris (ISBN 2-901057-37-3, 80-902487-0-5). 
Journees 1999 \& IX. Lohrmann-Colloquium, Motions of Celestial Bodies, Astrometry and Astronomical Reference Frames, Dresden, Germany, September 1999 (in preparation).

\section{REFERENES AND RELATED LINKS}

Commission 24: http://www.aip.de/groups/iau_c24/comm24.html Commission 8: http://www.ari.uni-heidelberg.de/interessantes/commission8 Division I: $\quad$ http://aries.usno.navy.mil/ad/iau.html

\section{Catalogues and Large Data Sets}

The Hipparcos and Tycho Catalogues: ESA (1997), ESA SP-1200 (ISBN 92-9092-399-7) The Hipparcos and Tycho Catalogues, Celestia 2000: ESA (1998), ESA SP-1220 (ISBN 92-9092-479-9)

AC 2000:

ACT Reference Catalogue: TRC:

USNO-A2:

SPM Catalog 2.0:

ARI CNS:

CdC Plate Archive:

VizieR catalogue database:

MAMA-Images: http://aries.usno.navy.mil/ad/ac/cite.html http://aries.usno.navy.mil/ad/act/act.html http://cdsweb.u-strasbg.fr/cgi-bin/Cat?I/250 http://aries.usno.navy.mil/ad/usnosa.html http://astro.yale.edu/astrom http://www. ari.uni-heidelberg.de/aricns http://webast.ast.obs-mip.fr/patrimoine/cdc_e.html http://vizir.u-strasbg.fr $h t t p: / / c d s w e b . u-s t r a s b g . f r$

\section{Measuring Machines}

APM: $\quad$ http://www.ast.cam.ac.uk/ apmcat

MAMA: http://dsmama.obspm.fr

PMM: $\quad h t t p: / / w w w . n o f s . n a v y . m i l / p r o j e c t s / p m m /$

Astrometric Space Missions

HIPPARCOS: http://astro.estec.esa.nl/SA-general/Projects/Hipparcos/hipparcos.html GAIA:

SIM:

FAME:

DIVA: http://astro.estec.esa.nl/SA-general/Projects/GAIA

http://sim.jpl.nasa.gov

http://aa.usno.navy.mil/FAME

http://www.aip.de/groups/DIVA

E. Schilbach

President of the Commission 